\title{
Efficacy and safety of endovascular thrombectomy in mild ischemic stroke: results from a retrospective study and meta-analysis of previous trials
}

Xian-Jin Shang ${ }^{1,2+}$, Zhong-Hua Shi ${ }^{3 \dagger}$, Cai-Feng He ${ }^{4+}$, Shuai Zhang ${ }^{1,5}$, Yong-Jie Bai ${ }^{1,6}$, Yong-Tao Guo ${ }^{1,7}$, Bo Sun ${ }^{1,7}$, Shun Li ${ }^{1,8}$, Huai-Ming Wang ${ }^{9}$, Zhi-Ming Zhou ${ }^{2}$, Wen-Jie Zi ${ }^{1,8^{*}}$ and Xin-Feng Liu ${ }^{1,8,9^{*}}$

\begin{abstract}
Background: Mechanical thrombectomy has been proven as a standard care for moderate to severe ischemic stroke with anterior large vessel occlusion (LVO); however, whether it is equally effective in mild ischemic stroke (MIS) is controversial.

Methods: In this retrospective study, a total of 177 Chinese patients presenting with MIS (NIHSS $\leq 8$ ) and LVO between January 2014 and September 2017 from seven comprehensive stroke centers were identified. Odds of good outcome with endovascular thrombectomy versus medical treatment were obtained by logistic regression analysis and propensity-score matching method, and a meta-analysis pooled results from six studies $(n=733)$.

Results: Good outcome (mRS: 0-1) was 58.2\% (46/79) in the thrombectomy and 46.9\% (46/98) in the medical group, which showed no statistical significance before adjustment $(P=0.13 ; \mathrm{OR}=1.57,95 \% \mathrm{Cl}: 0.86$ to 2.86$)$. The adjusted ORs of thrombectomy versus medical group were $3.23(95 \% \mathrm{Cl}, 1.35$ to $7.73 ; P=0.008)$ by multivariable logistic analysis, $2.78(1.12$ to $6.89 ; P=0.02)$ by propensity score matching analysis, and $3.20(1.22$ to $8.37 ; P=0.01)$ by propensity score matching analysis with additional adjustments, respectively. Thrombectomy treatment did not result in excessive mortality or symptomatic intracranial hemorrhage after adjustments. The meta-analysis did not confirm the associations between good outcome and endovascular treatment.
\end{abstract}

Conclusions: The current study indicates that endovascular thrombectomy is associated with good functional outcome in MIS patients with LVO, and without additional risk of symptomatic intracranial hemorrhage and mortality. Although the meta-analysis failed to demonstrate its superiority compared to medical treatment, randomized clinical trials are needed.

Keywords: Thrombectomy, Stroke, Endovascular, Outcome

\section{Background}

Since the publication of six randomized control trials, mechanical endovascular thrombectomy (MET) has become a standard therapy for patients with moderate to severe ischemic stroke and large vessel occlusion (LVO) in the proximal anterior circulation [1-6]. Mild ischemic

\footnotetext{
*Correspondence: ziwenjie1981@163.com; xfliu2@vip.163.com

${ }^{\dagger}$ Xian-Jin Shang, Zhong-Hua Shi and Cai-Feng He contributed equally and are co-first authors

'Department of Neurology, Jinling Hospital, Jinling Clinical College of

Nanjing Medical University, Nanjing 210002, Jiangsu, China

Full list of author information is available at the end of the article
}

stroke (MIS) accounts for $>15 \%$ of acute ischemic stroke, and patients with LVO also have a risk of severe deterioration if there is no reperfusion [7-9]. Endovascular therapy was reported to rapidly and effectively recanalize the occluded vessel, but also increased the risk of intracranial hemorrhage associated with no improvement of clinical functional outcome [10, 11]. Conversely, endovascular treatment was reported to improve the clinical symptoms of patients with MIS during hospitalization, and also improve long-term prognosis

C The Author(s). 2019 Open Access This article is distributed under the terms of the Creative Commons Attribution 4.0 International License (http://creativecommons.org/licenses/by/4.0/), which permits unrestricted use, distribution, and 
[12]. Therefore, the efficacy and safety of MET for MIS remains controversial.

In this study, we retrospectively analyzed the data of MIS patients treated with MET or medical therapy from our center, and combined the previously published data through a meta-analysis to obtain more reliable conclusions.

\section{Methods}

\section{Patient selection}

We conducted a retrospective study involving patients with acute ischemic stroke who were consecutively admitted to 7 comprehensive stroke centers from China (Jinling Hospital, Yijishan Hospital, Fuzhou General Hospital of Nanjing Military Region, Affiliated Hospital of Yangzhou University, Daping Hospital, Hubei Zhongshan Hospital, and No.123 Hospital of the People's Liberation Army) between January 2014 and August 2017, and all these patients initially presented with mild neurological deficit during $24 \mathrm{~h}$, which defined as NIHSS $\leq 8$ (because the cut-off of 8 always recognized as probably having LVO), and was diagnosed as having LVO, including ICA, M1 or M2 segment of MCA, and ACA on CTA or MRA. The specific exclusion criteria were: (i) admission age $<18$ years, (ii) patients who had a prior modified Rankin Score (mRS) $\geq 2$, and (iii) arteriovenous malformation and arterial aneurysm determined by CTA/MRA.

All eligible subjects were divided into the MET group or the medical group, according to whether MET was performed. The MET group included the initial MET and rescue MET [13]. The local Ethics Committees of each center approved the use of patients' data for this study, and written consents of the patients were waived due to its retrospective nature.

\section{Data collection}

We retrieved demographic, clinical, and neuroimaging data from all eligible subjects, including age, sex, stroke risk factors (atrial fibrillation, hypertension, dyslipidemia, diabetes and smoking), admission systolic blood pressure, admission NIHSS scores, time of onset to imaging, use of intravenous thrombolysis, and the ASPECT score and collateral circulation assessment based on admission imaging data. Good collateral circulation indicated rapid or complete collateral collateral flow into the ischemic area. Stroke etiology was defined according to the Trial of Org 10,172 in Acute Stroke Treatment classification, and was grouped into large atherosclerosis, cardioembolism, and others/undetermined [14]. Stroke occlusion location was divided into ICA, MCA (M1 or M2 segments), and tandem occlusion corresponding to the artery occlusion occurring in more than two different parts of a continuous vessel.
All radiological data and clinical medical records of the subjects were sent to the core laboratory in our hospital, and were reviewed in a blinded fashion by two neurologists (Y-T Guo and S Zhang) with advice of a third experienced neurologist (W-J $\mathrm{Zi}$ ) in cases of disagreement.

\section{Clinical outcomes}

The patients' functional outcomes at 3 months were measured by the mRS, ranging from 0 to 6 (higher scores indicate more severe disability), which was collected by telephone follow-up or outpatient visit in each center by staff with a structured interview [15]. Symptomatic intracranial hemorrhage $(\mathrm{sICH})$ was considered as any hemorrhage combined with an increase of $\geq 4$ points in total NIHSS score, or $\geq 2$ points in one NIHSS category, according to the Heidelberg classification scheme [16]. The primary outcome of this study was good outcome defined as an mRS $0-1$, while secondary outcomes included favorable outcome as mRS 0-2, mortality at 3 months and sICH at $48 \mathrm{~h}$.

\section{Meta-analysis}

A pooled analysis of studies comparing endovascular with medical treatment, either with or without intravenous tissue plasminogen activator, in patients with mild stroke and LVO was conducted. We retrieved the relevant studies from four electronic databases including Pubmed, Embase, Cochrane Database of Systematic Reviews and Cochrane Central Register of Controlled Trials from database inception to May 2018, and used the appropriate free text and Mesh terms to identify them: "mild stroke", "minor stroke", "minimal stroke", "NIHSS $\leq 8$ ", and "recanalization", "endovascular", "thrombectomy", "stent-retriever", "thrombolysis", and "reperfusion". Additional records were identified through reference lists of eligible studies.

All the retrieved studies were screened by two independent reviewers (X-J Shang and C-F He) according to the following criteria: (i) MIS patients with LVO (ICA, MCA M1 and M2, ACA, posterior circulation), defined as baseline NIHSS $\leq 8$ at onset, (ii) all studies selected included endovascular or thrombectomy treatment, and case reports, abstracts, editorials and expert opinions were excluded, and (iii) all studies selected should be human trials and in English, and if more than one trials came from the same center with the same dataset, only the most complete dataset published was used for final analysis. The third reviewer (W-J Zi) assessed the quality of observational trials and cohort studies using the Newcastle-Ottawa scale, and coordinated and resolved the discrepancies between reviewers. 


\section{Statistical analysis}

Qualitative variables were shown as number and percentage, and for quantitative variables, normally distributed data were presented as mean and standard deviation, while non-normally distributed data were presented as median and interquartile range. The difference in baseline characteristics between MET group and medical group was assessed using calculating the absolute standardized difference (ASD), and ASD > 0.10 was recognized as significant difference.

We adopted the following strategies to compare clinical outcomes between the two groups: (i) Multivariable logistic regression was performed using variables with ASD $>0.10$ on comparisons of baseline characteristics between the two groups; (ii) Propensity score (PS) matching method was used to reduce the effects of potential confounding factors on between-group comparisons, and calculated the odds ratio (OR) for the MET versus medical therapy as the treatment effect size. (iii) If the baseline differences still existed after the PS matching, we included variable factors with ASD $>0.10$ into the logistic regression equation to further calculate the treatment effect size.

In the meta-analysis, the possible clinical or methodological variation had been taken into account, so we adopted a randomized model. We used $\chi^{2}$ tests to assess heterogeneity between trials and $\mathrm{I}^{2}$ statistic to estimate the percentage of total variation across studies, with values beyond $50 \%$ regarded as substantial heterogeneity.

Significance was set at $P<0.05$ using two-sided tests. All statistical analyses were performed with Statistical Software (IBM SPSS Statistics 22; IBMArmonk, New York, USA), and Review Manager Version 5.3.4 (Cochrane Collaboration, Software Update, Oxford, United Kingdom).

\section{Results \\ Baseline characteristics}

A total of 79 patients treated with MET and 98 patients having medical treatment were identified in this study. The Table 1 shows the baseline characteristics of two groups before and after PS-matching. Most of the variables had substantial differences (ASD >0.10), except for the hypertension history and ASPECTS before matching. These differences were reduced after matching, with an ASD $>0.10$ only for sex, atrial fibrillation, intravenous thrombolysis and stroke etiology.

\section{Clinical outcomes}

There was no difference in crude comparison of primary outcome (mRS 0-1) between MET and medical group (58.2\% versus $46.9 \% ; P=0.13$ ); of the secondary outcome, MET group had more substantial sICH risks (10.1\% versus $2.0 \%$; OR, 95\% confidence interval [CI]:
5.4, [1.11, 26.24]; $P=0.02$ ), but favorable outcome (mRS $0-2$ : $70.9 \%$ versus $59.2 \% ; P=0.10)$ and mortality at 3 months $(5.1 \%$ versus $1.0 \% ; P=0.17)$ did not show substantial difference. However, multivariable logistic regression analyses showed a statistically significant association of MET with good outcome (OR, 95\% CI: 3.23, $[1.35,7.73] ; P=0.008)$ and favorable outcome (OR, 95\% CI: 2.59, [1.06, 6.33]; $P=0.03)$, and not with a higher sICH risk (OR, 95\%CI: 3.05, [0.44, 21.23]; $P=$ 0.25). After the PS matching, the effect of MET on good outcome remained significant (OR, 95\%CI: 2.78, [1.12, 6.89]; $P=0.02$ ), and additional adjustment for variables with ASD > 0.10 did not affect the statistical significance of this association (OR, 95\%CI: 3.20, [1.22, 8.37]; $P=$ 0.01). (See Table 2 and Fig. 1).

\section{Meta-analysis}

Of the 2484 records retrieved through database screening and other resources, 5 studies (this study named 'Shang 2018') were included in the final analysis (see in Additional file 1: Figure S1). [8, 10, 11, 17, 18] A total of 733 cases were included in the studies, 226 of which were comprised of 113 matched pairs. The analysis was conducted according to the NIHSS cutoff 8 or 5 , and to compare endovascular treatment and medical therapy irrespective of rt-PA. Two studies were included in the comparison of MET versus medical treated patients with NIHSS $\leq 8$ and LVO. When compared to patients who did not receive endovascular recanalization, patients treated with medical therapy had the similar functional outcome and procedural complications. (see Additional file 1: Figures S2 and S3). Four studies compared the clinical outcomes of patients with NIHSS $\leq 5$ subgroup, the meta-analysis failed to find a correlation between endovascular treatment and clinical outcomes (see Additional file 1: Figures S4 and S5). All tests for subgroup difference did not find substantial heterogeneity.

\section{Discussion}

In the present study, we found that endovascular thrombectomy was effective for improving good functional outcome, although it appeared to increase the incidence of symptomatic intracerebral bleeding, for stroke patients with mild deficits and proximal anterior LVO.

Mild stroke patients with LVO showed a worse prognosis and higher mortality compared with stroke patients without evidence of LVO. [19, 20] Thus, it was suggested that mild stroke patients presenting with proximal arterial occlusion should not be considered as MIS. [21] In a study of 204 eligible mild stroke patients with proximal LVO who did not receive any recanalization approaches, Mokin et al. reported that only $62 \%$ of patients were able to 
Table 1 Baseline characteristics of mild stroke patients according to treatment approach before and after propensity score-matching

\begin{tabular}{|c|c|c|c|c|c|c|}
\hline & \multicolumn{3}{|c|}{ Before Propensity Score-matching } & \multicolumn{3}{|c|}{ After Propensity Score-matching } \\
\hline & MET group & Medical group & ASD & MET group & Medical group & ASD \\
\hline Number & 79 & 98 & - & 40 & 40 & - \\
\hline Age (years), mean $\pm S D$ & $61.2 \pm 14.4$ & $65.9 \pm 10.7$ & 0.323 & $65.1 \pm 11.6$ & $65.0 \pm 10.5$ & 0.005 \\
\hline Sex (male) & $52(65.8)$ & $72(73.5)$ & 0.160 & $27(67.5)$ & $29(72.5)$ & 0.105 \\
\hline \multicolumn{7}{|l|}{ Vascular risk factors } \\
\hline Atrial fibrillation & $21(26.6)$ & $13(13.3)$ & 0.300 & $9(22.5)$ & $7(17.5)$ & 0.112 \\
\hline Hypertension & $51(64.6)$ & $62(63.3)$ & 0.027 & $28(70.0)$ & $28(70.0)$ & NA \\
\hline Hyperlipidemia & $10(12.7)$ & $19(19.4)$ & 0.201 & $6(15.0)$ & $5(12.5)$ & 0.075 \\
\hline Diabetes & $11(13.9)$ & 31 (31.6) & 0.508 & $10(25.0)$ & $11(27.5)$ & 0.072 \\
\hline Smoking & $17(21.5)$ & $34(34.7)$ & 0.319 & $8(20.0)$ & $9(22.5)$ & 0.006 \\
\hline \multicolumn{7}{|l|}{ Basement measurements } \\
\hline Systolic blood pressure, mean \pm SD & $139.4 \pm 20.9$ & $147.0 \pm 23.8$ & 0.361 & $144.7 \pm 23.7$ & $143.4 \pm 21.5$ & 0.006 \\
\hline Admission NIHSS, median (IQR) & $7(5-8)$ & $4(2-6)$ & 0.868 & $5.5(4-7)$ & $5.5(4-7)$ & NA \\
\hline ASPECTS, median (IQR) & $9(8-10)^{a}$ & $9(8-10)$ & 0.098 & $9(8-10)$ & $10(8.25-10)$ & 0.033 \\
\hline Good collateral circulation ${ }^{\text {b }}$ & $54(68.4)$ & $70(71.4)$ & 0.066 & $27(67.5)$ & $26(65.0)$ & 0.052 \\
\hline Onset to imaging (min), median (IQR) & $300(215-390)$ & $300(180-480)$ & 0.432 & $284(225-386)$ & $240(180-360)$ & 0.057 \\
\hline Onset to puncture (min), mean (range) & $314(10-995)$ & NA & NA & $284(10-758)$ & NA & NA \\
\hline Puncture to reperfusion (min),median (IQR) & 75 (54-95) & NA & NA & $75(55-93)$ & NA & NA \\
\hline Intravenous thrombolysis & $23(29.1)$ & $11(11.2)$ & 0.391 & $13(32.5)$ & $10(25.0)$ & 0.164 \\
\hline \multicolumn{7}{|l|}{ Etiology } \\
\hline Large artery atherosclerosis & $41(51.9)$ & 85 (86.7) & 0.693 & $29(72.5)$ & $31(77.5)$ & 0.099 \\
\hline Embolism & $29(36.7)$ & $11(11.2)$ & 0.525 & $9(22.5)$ & $7(17.5)$ & 0.103 \\
\hline Others/undetermined & $9(11.4)^{c}$ & $2(2.0)$ & 0.292 & $2(5.0)$ & $2(5.0)$ & NA \\
\hline \multicolumn{7}{|l|}{ Site of occlusion } \\
\hline ICA & $5(6.3)$ & $25(25.5)$ & 0.400 & $4(10.0)$ & $5(12.5)$ & 0.069 \\
\hline MCA M1 & $49(62.0)$ & 39 (39.8) & 0.455 & $21(52.5)$ & $21(52.5)$ & NA \\
\hline MCA M2 & $12(15.2)$ & $14(14.3)$ & 0.025 & $7(17.5)$ & $6(15.0)$ & 0.069 \\
\hline Tandem & $13(16.5)$ & $20(20.4)$ & 0.106 & $8(20.0)$ & $8(20.0)$ & NA \\
\hline
\end{tabular}

Value were showed as $\mathrm{n}(\%)$ if not mentioned

ASD absolute standardized difference, IQR interquartile range, SD standard deviation, NA not applicable

${ }^{a}$ Two missing value were replaced by median; 'dissection (2 cases), thrombophilia (2 cases), cryptogenic stroke (5 cases)

${ }^{\mathrm{b}}$ MET group was assessed according to digital subtraction angiogram, while medical group according to MR/CT resource angiogram

ambulate independently (mRS $0-3)$ at discharge. [22] By contrast, in the present study of a similar cohort of patients treated with endovascular thrombectomy, $70.9 \%$ exhibited functional independence (mRS 0-2) at 3 months, $10.1 \%$ showed sICH, and $5.1 \%$ all-cause mortality. In support, in a subset of stroke patients (NIHSS < 8 ) treated with MET, Dargazanli et al. reported $78.3 \%$ for 3-month favorable outcome and $5.1 \%$ for mortality. [23] Furthermore, in MET treated patients with an NIHSS $\leq 8$, Pfaff et al. reported $63.6 \%$ for favorable outcome, $6.1 \%$ for $\mathrm{sICH}$, and 9.1\% for death. [24] Thus, overall these studies support that endovascular treatment for MIS and proximal LVO is favorably effective, as observed for intravenous thrombolysis. [25]
However, the proven evidence for the efficacy of thrombectomy therapy in MIS is still not available, and there is considerable heterogeneity among studies. For example, a pooled analysis from five recent randomized trials reported a negative finding in patients with an NIHSS $\leq 10$ and LVO for thrombectomy compared with medical treatment. [26] Similarly, in a recent, larger multicenter intention-to-treat cohort study in MIS patients with an NIHSS $<8$, there was no consistent improvement in good and favorable outcome. [8] As a result, further randomized controlled trials are needed to solve the uncertainty.

The main limitation of our study was the nonrandomized design, and the limited number of patients may have underpowered our interpretation. 
Table 2 Odds ratios for clinical outcome by mechanical thrombectomy compared to medical therapy

\begin{tabular}{|c|c|c|c|c|c|c|c|c|c|c|c|c|}
\hline & \multicolumn{3}{|c|}{ mRS $0-1$ at 3 months } & \multicolumn{3}{|c|}{ mRS $0-2$ at 3 months } & \multicolumn{3}{|c|}{$\mathrm{slCH}$ at $48 \mathrm{~h}$} & \multicolumn{3}{|c|}{ Mortality at 3 months } \\
\hline & $\overline{\mathrm{OR}}$ & $95 \% \mathrm{Cl}$ & $P$ value & $\overline{\mathrm{OR}}$ & $95 \% \mathrm{Cl}$ & $P$ value & $\overline{\mathrm{OR}}$ & $95 \% \mathrm{Cl}$ & $P$ value & $\overline{\mathrm{OR}}$ & $95 \% \mathrm{Cl}$ & $P$ value \\
\hline Crude analysis & 1.57 & 0.86 to 2.86 & 0.13 & 1.67 & 0.89 to 3.15 & 0.10 & 5.40 & 1.11 to 26.24 & 0.02 & 5.17 & 0.56 to 47.25 & 0.17 \\
\hline Multivariable analysis ${ }^{a}$ & 3.23 & 1.35 to 7.73 & 0.008 & 2.59 & 1.06 to 6.33 & 0.03 & 3.05 & 0.44 to 21.23 & 0.25 & 2.19 & 0.13 to 37.08 & 0.58 \\
\hline PS matching ${ }^{b}$ & 2.78 & 1.12 to 6.89 & 0.02 & 1.85 & 0.75 to 4.55 & 0.17 & 2.71 & 0.49 to 14.90 & 0.43 & 4.33 & 0.46 to 40.60 & 0.35 \\
\hline $\begin{array}{l}\text { PS matching with } \\
\text { additional adjustments }\end{array}$ & 3.20 & 1.22 to 8.37 & 0.01 & 2.08 & 0.79 to 5.47 & 0.13 & 3.08 & 0.45 to 20.69 & 0.24 & 4.85 & 0.48 to 49.04 & 0.18 \\
\hline
\end{tabular}

Cl confidence interval, OR Odd Ratio, PS propensity score

${ }^{a}$ Adjusted for age, sex, medical histories (atrial fibrillation, hyperlipidemia, diabetes), smoking, systolic blood pressure, admission NIHSS, time of onset to imaging, intravenous thrombolysis, stroke etiology and site of occlusion

${ }^{b}$ PS-matched sample included 40 pairs with 1:1 ratio

'Adjusted for Sex, atrial fibrillation, intravenous thrombolysis and stroke etiology in mRS outcome and mortality; adjusted for age, sex, atrial fibrillation, diabetes,

intravenous thrombolysis, ASPECTS, time of onset to imaging and stroke etiology in SICH

Also due to the small sample size in this study, we did not further present the thrombectomy prognosis of patients with NIHSS $\leq 5$, which was widely recognized as mild ischemic stroke, although the primary outcome was still positive.

As a highlight of this study, we focused on the metaanalysis of the efficacy and safety of endovascular treatment for mild stroke, and found no substantial differences of endovascular versus medical treatment for patients with NIHSS 8 or 5 on matching or not. The specific reasons of inconsistence with our data are as follows: first of all, all studies diagnostic criteria were not completely consistent, e.g. some studies recruited cases with the posterior circulation infarction, and it is well known that these patients might progress rapidly and had poorer outcome compared with those in anterior circulation, which could have an effect on the outcomes. Also, the definition criterion of symptomatic bleeding was variable, and there were different endovascular procedures between studies, including intraarterial thrombolysis, angioplasty and mostly thrombectomy, which might confuse the practical effects of thrombectomy. Therefore, it is recommended to obtain more detailed information of other studies and

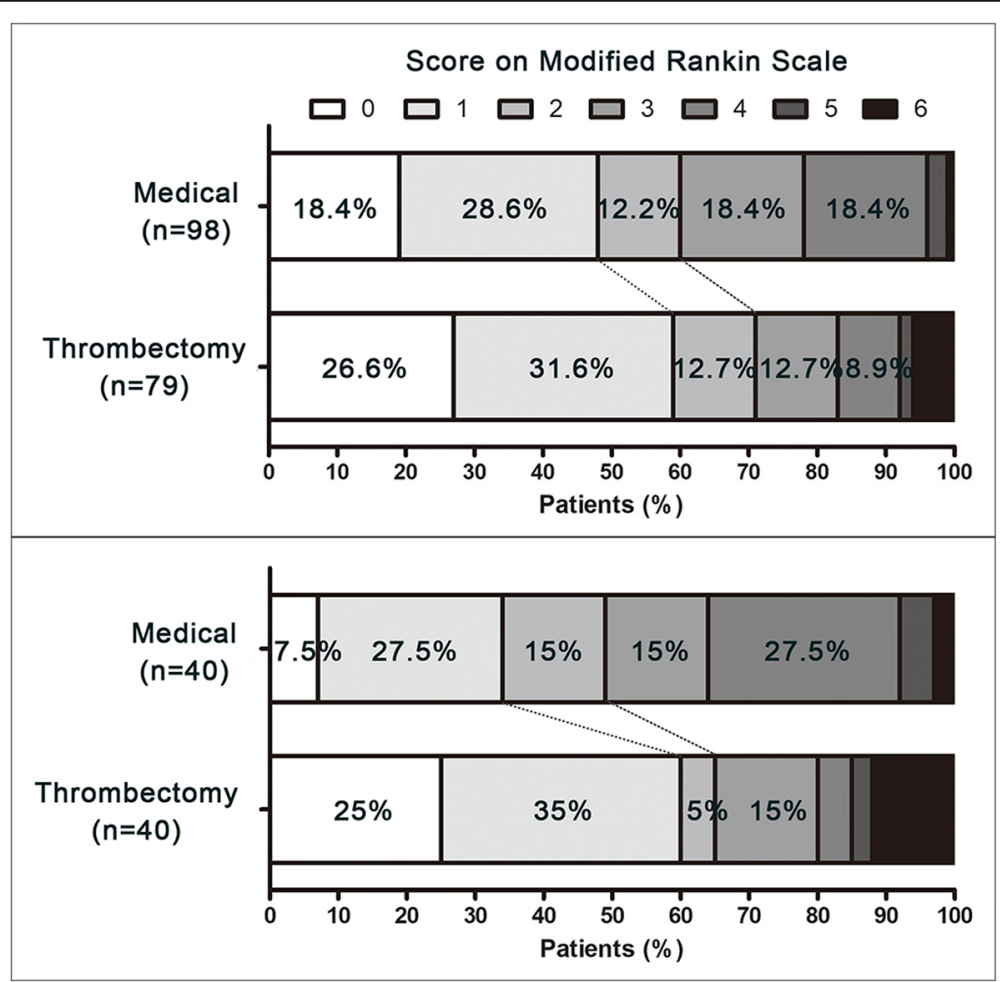

Fig. 1 mRS at 3 months follow-up of stroke patients treated by thrombectomy and medical approach 
stratify the meta-analysis, which may be accessible to original nature.

\section{Conclusions}

Our data have found a statistically significant benefit of endovascular thrombectomy for MIS patients with proximal LVO in anterior circulation, but it should be confirmed by further high quality trials.

\section{Additional files}

Additional file 1: Figure S1. PRISMA (Preferred Reporting Items for Systematic Reviews and Meta-Analyses) flow-chart of search strategy. Figure S2. Forest plot of outcomes between endovascular treatment and medical therapy in patients with NIHSS $\leq 8$. Figure S3. Forest plot of outcomes between endovascular treatment and medical therapy in matched patients with NIHSS $\leq 8$. Figure S4. Forest plot of outcomes between endovascular treatment and medical therapy in patients with $\mathrm{NIHSS} \leq 5$. Figure S5. Forest plot of outcomes between endovascular treatment and medical therapy in matched patients with NIHSS $\leq 5$. (DOCX $2 \mathrm{~kb})$

\section{Abbreviations}

ASPECTS: Alberta stroke program early CT score; Cl: Confidence interval; CTA: computed tomography angiography; ICA: Intracranial carotid artery; LVO: Large vessel occlusion; MCA: Middle cerebral artery; MIS: Mild ischemic stroke; MRA: Magnetic resonance angiography; mRS: modified Rankin Scale; NIHSS: National institute of health stroke scale; OR: Odds ratio;

$\mathrm{RCT}$ : Randomized control trials; $\mathrm{SICH}$ : symptomatic intracranial hemorrhage

\section{Acknowledgments}

We thank to all participants and engaging centers.

\section{Authors' contributions}

XJS, ZHS: conception and design, analysis and interpretation of data and drafting the article; CFH: design and analysis of meta-analysis, revising it critically for important intellectual content; SZ, YJB, YTG, BS, SL, HMW and ZMZ: acquisition of data, revising it critically for important intellectual content; XFL and WJZ: conception and design, analysis and interpretation of data and revising it critically for important intellectual content. All authors read and approved the final manuscript.

\section{Funding}

This work was supported by National Natural Science Foundation of China grant number (81530038, 81400993, 81671172 and 81701184), National Key Research and Development Program grant number (No.2017YFC1307901), Chinese Postdoctoral Science Fund grant number (2015 M572815).

\section{Availability of data and materials}

The data is available on request to the corresponding author.

\section{Ethics approval and consent to participate}

The local Ethics Committees of each center (Jinling Hospital, Yijishan Hospital, Fuzhou General Hospital of Nanjing Military Region, Affiliated Hospital of Yangzhou University, Daping Hospital, Hubei Zhongshan Hospital, and No.123 Hospital of the People's Liberation Army) approved the use of patients' data for this study, and written consents of the patients were waived due to its retrospective nature.

\section{Consent for publication}

Not applicable.

\section{Competing interests}

The authors declare that they have no competing interests.

\section{Author details}

'Department of Neurology, Jinling Hospital, Jinling Clinical College of Nanjing Medical University, Nanjing 210002, Jiangsu, China. ${ }^{2}$ Department of
Neurology, Yijishan Hospital of Wannan Medical College, Wuhu 241001, Anhui, China. ${ }^{3}$ Department of Neurosurgery, The 101st Hospital of the People's Liberation Army, Wuxi 214000, Jiangsu, China. ${ }^{4}$ Department of Dermatology, Yijishan Hospital of Wannan Medical College, Wuhu 241001, Anhui, China. ${ }^{5}$ Department of Neurology, The affiliated Hospital of Yangzhou University, Yangzhou 225001, Jiangsu, China. ${ }^{6}$ Department of Neurology, First Affiliated Hospital, and College of Clinical Medicine of Henan University of Science and Technology, Luoyang 471003, China. ${ }^{7}$ Department of Neurology, The Affiliated Huai'an NO.1 People's Hospital, Nanjing Medical University, Huai'an 223300, Jiangsu, China. ${ }^{8}$ Department of Neurology, Jinling Hospital, Southern Medical University, Nanjing 210002, Jiangsu, China. ${ }^{9}$ Department of Neurology, Jinling Hospital, Medical School of Nanjing University, Nanjing 210002, Jiangsu, China.

Received: 20 July 2018 Accepted: 19 June 2019

Published online: 05 July 2019

\section{References}

1. Berkhemer OA, Fransen PS, Beumer D, van den Berg LA, Lingsma HF, Yoo AJ, et al. A randomized trial of intraarterial treatment for acute ischemic stroke. N Engl J Med. 2015;372:11-20.

2. Campbell BC, Mitchell PJ, Kleinig TJ, Dewey HM, Churilov L, Yassi N, et al. Endovascular therapy for ischemic stroke with perfusion-imaging selection. N Engl J Med. 2015;372:1009-18.

3. Goyal M, Demchuk AM, Menon BK, Eesa M, Rempel JL, Thornton J, et al. Randomized assessment of rapid endovascular treatment of ischemic stroke. N Engl J Med. 2015;372:1019-30.

4. Jovin TG, Chamorro A, Cobo E, de Miquel MA, Molina CA, Rovira A, et al. Thrombectomy within 8 hours after symptom onset in ischemic stroke. N Engl J Med. 2015;372:2296-306.

5. Saver JL, Goyal M, Bonafe A, Diener HC, Levy El, Pereira VM, et al. Stentretriever thrombectomy after intravenous t-PA vs. t-PA alone in stroke. N Engl J Med. 2015;372:2285-95.

6. Bracard S, Ducrocq X, Mas JL, Soudant M, Oppenheim C, Moulin T, et al. Mechanical thrombectomy after intravenous alteplase versus alteplase alone after stroke (THRACE): a randomised controlled trial. Lancet Neurol. 2016;15: $1138-47$.

7. Heldner MR, Jung S, Zubler C, Mordasini P, Weck A, Mono ML, et al. Outcome of patients with occlusions of the internal carotid artery or the main stem of the middle cerebral artery with NIHSS score of less than 5: comparison between thrombolysed and non-thrombolysed patients. J Neurol Neurosurg Psychiatry. 2015;86:755-60.

8. Dargazanli C, Arquizan C, Gory B, Consoli A, Labreuche J, Redjem H, et al. Mechanical Thrombectomy for minor and mild stroke patients harboring large vessel occlusion in the anterior circulation: a multicenter cohort study. Stroke. 2017:48:3274-81.

9. Heldner MR, Zubler C, Mattle HP, Schroth G, Weck A, Mono ML, et al. National Institutes of Health stroke scale score and vessel occlusion in 2152 patients with acute ischemic stroke. Stroke. 2013;44:1153-7.

10. Messer MP, Schonenberger S, Mohlenbruch MA, Pfaff J, Herweh C, Ringleb PA, et al. Minor stroke syndromes in large-vessel occlusions: mechanical thrombectomy or thrombolysis only? AJNR Am J Neuroradiol. 2017;38:1177-9.

11. Urra X, San Roman L, Gil F, Millan M, Canovas D, Roquer J, et al. Medical and endovascular treatment of patients with large vessel occlusion presenting with mild symptoms: an observational multicenter study. Cerebrovasc Dis. 2014;38:418-24.

12. Haussen DC, Bouslama M, Grossberg JA, Anderson A, Belagage S, Frankel M, et al. Too good to intervene? Thrombectomy for large vessel occlusion strokes with minimal symptoms: an intention-to-treat analysis. J Neurointerv Surg. 2017;9:917-21.

13. Shang $X$, Lin M, Zhang S, Li S, Guo Y, Wang W, et al. Clinical outcomes of endovascular treatment within 24 hours in patients with mild ischemic stroke and perfusion imaging selection. AJNR Am J Neuroradiol. 2018;39: 1083-7.

14. Adams HP Jr, Bendixen BH, Kappelle LJ, Biller J, Love BB, Gordon DL, et al. Classification of subtype of acute ischemic stroke. Definitions for use in a multicenter clinical trial. TOAST. Trial of org 10172 in acute stroke treatment. Stroke. 1993;24:35-41.

15. Wilson JT, Hareendran A, Grant M, Baird T, Schulz UG, Muir KW, et al. Improving the assessment of outcomes in stroke: use of a structured 
interview to assign grades on the modified rankin scale. Stroke. 2002;33: 2243-6.

16. von Kummer R, Broderick JP, Campbell BC, Demchuk A, Goyal M, Hill MD, et al. The Heidelberg bleeding classification: classification of bleeding events after ischemic stroke and reperfusion therapy. Stroke. 2015;46:2981-6.

17. Haussen DC, Lima FO, Bouslama M, Grossberg JA, Silva GS, Lev MH, et al. Thrombectomy versus medical management for large vessel occlusion strokes with minimal symptoms: an analysis from STOPStroke and GESTOR cohorts. J Neurointerv Surg. 2018;10:325-9.

18. Cerejo R, Cheng-Ching E, Hui F, Hussain MS, Uchino K, Bullen J, et al. Treatment of patients with mild acute ischemic stroke and associated large vessel occlusion. J Clin Neurosci. 2016;30:60-4

19. Nedeltchev K, Schwegler B, Haefeli T, Brekenfeld C, Gralla J, Fischer U, et al. Outcome of stroke with mild or rapidly improving symptoms. Stroke. 2007; 38:2531-5.

20. Zhu W, Churilov L, Campbell BC, Lin M, Liu X, Davis SM, et al. Does large vessel occlusion affect clinical outcome in stroke with mild neurologic deficits after intravenous thrombolysis? J Stroke Cerebrovasc Dis. 2014;23: 2888-93.

21. Kim JT, Park MS, Chang J, Lee JS, Choi KH, Cho KH. Proximal arterial occlusion in acute ischemic stroke with low NIHSS scores should not be considered as mild stroke. PLoS One. 2013:8:e70996.

22. Mokin M, Masud MW, Dumont TM, Ahmad G, Kass-Hout T, Snyder KV, et al. Outcomes in patients with acute ischemic stroke from proximal intracranial vessel occlusion and NIHSS score below 8. J Neurointerv Surg. 2014;6:413-7.

23. Dargazanli C, Consoli A, Gory B, Blanc R, Labreuche J, Preda C, et al. Is reperfusion useful in Ischaemic stroke patients presenting with a low National Institutes of Health stroke scale and a proximal large vessel occlusion of the anterior circulation? Cerebrovasc Dis. 2017:43:305-12.

24. Pfaff J, Herweh C, Pham M, Schonenberger S, Nagel S, Ringleb PA, et al. Mechanical Thrombectomy in patients with acute ischemic stroke and lower NIHSS scores: recanalization rates, Periprocedural complications, and clinical outcome. AJNR Am J Neuroradiol. 2016;37:2066-71.

25. Laurencin C, Philippeau F, Blanc-Lasserre K, Vallet AE, Cakmak S, Mechtouff $L$, et al. Thrombolysis for acute minor stroke: outcome and barriers to management. Results from the RESUVAL stroke network. Cerebrovasc Dis. 2015:40:3-9.

26. Goyal M, Menon BK, van Zwam WH, Dippel DW, Mitchell PJ, Demchuk AM, et al. Endovascular thrombectomy after large-vessel ischaemic stroke: a meta-analysis of individual patient data from five randomised trials. Lancet. 2016;387:1723-31.

\section{Publisher's Note}

Springer Nature remains neutral with regard to jurisdictional claims in published maps and institutional affiliations.

Ready to submit your research? Choose BMC and benefit from:

- fast, convenient online submission

- thorough peer review by experienced researchers in your field

- rapid publication on acceptance

- support for research data, including large and complex data types

- gold Open Access which fosters wider collaboration and increased citations

- maximum visibility for your research: over $100 \mathrm{M}$ website views per year

At $\mathrm{BMC}$, research is always in progress.

Learn more biomedcentral.com/submissions 